\title{
CLASSIFICATIONS OF ROTATION SURFACES IN PSEUDO-EUCLIDEAN SPACE
}

\author{
Young Ho KIM AND DaE WON YOON
}

\begin{abstract}
In this article, we study rotation surfaces in the 4dimensional pseudo-Euclidean space $\mathbb{E}_{2}^{4}$. Also, we obtain the complete classification theorems for the flat rotation surfaces with finite type Gauss map, pointwise 1-type Gauss map and an equation in terms of the mean curvature vector. In fact, we characterize the flat rotation surfaces of finite type immersion with the Gauss map and the mean curvature vector field, namely the Gauss map of finite type, pointwise 1-type Gauss map and some algebraic equations in terms of the Gauss map and the mean curvature vector field related to the Laplacian of the surfaces with respect to the induced metric.
\end{abstract}

\section{Introduction}

A pseudo-Riemannian submanifold $M$ of the $m$-dimensional pseudoEuclidean space $\mathbb{E}_{s}^{m}$ with signature $(s, m-s)$ is said to be of finite type if its position vector field $x$ can be expressed as a finite sum of eigenvectors of the Laplacian $\Delta$ of $M$, that is, $x=x_{0}+\sum_{i=1}^{k} x_{i}$, where $x_{0}$ is a constant map, $x_{1}, \ldots, x_{k}$ non-constant maps such that $\Delta x_{i}=$ $\lambda_{i} x_{i}, \lambda_{i} \in \mathbb{R}, i=1,2, \ldots, k$. If $\lambda_{1}, \lambda_{2}, \ldots, \lambda_{k}$ are different, then $M$ is said to be of $k$-type. Similarly, a smooth map $\phi$ of an $n$-dimensional pseudoRiemannian submanifold $M$ of $\mathbb{E}_{s}^{m}$ is said to be of finite type if $\phi$ is a finite sum of $\mathbb{E}_{s}^{m}$ - valued eigenfunctions of $\Delta$. As is seen in the recent publication, for example, [3], [7], [12], [16], [19], [21], [23] and [24], the notion of finite type Gauss map is a especially useful tool in the study of submanifolds. Also, it is the natural extension of the study of minimal submanifolds on which many mathematicians have devoted in the last

Received February 14, 2003.

2000 Mathematics Subject Classification: 53B25, 53C50.

Key words and phrases: rotation surfaces, Gauss map, finite type, Pseudo-Euclidean space.

This work was supported by KOSEF research project No. 2001-1-10200-006-2. 
years. The first results on this subject have been collected in book ([8]); for a recent survey, see [9]. Many works were done to characterize or classify submanifolds in terms of finite type. In a framework of the theory of finite type, B.-Y. Chen and P. Piccini ([12]) made a general study on compact submanifolds of Euclidean spaces with finite type Gauss map.

It is interesting to ask which submanifolds with finite type Gauss map are themselves of finite type. In [3] C. Baikoussis, B.-Y. Chen and L. Verstraelen classified ruled surfaces with finite type Gauss map in Euclidean $m$-space $\mathbb{E}^{m}$. Furthermore, in [21] the authors investigated the finite type Gauss map of the ruled surfaces with non-null base curve in an $m$-dimensional Minkowski space $\mathbb{E}_{1}^{m}$, and in [20] D.- S. Kim and the authors completely classified ruled surfaces with 1-type Gauss map in the $m$-dimensional Minkowski spaces $\mathbb{E}_{1}^{m}$.

If a submanifold $M$ of a Euclidean space or a pseudo-Euclidean space has 1-type Gauss map $G$, then $G$ satisfies $\Delta G=\lambda(G+C)$ for some $\lambda \in \mathbb{R}$ and some constant vector $C$. However, the Laplacian of the Gauss map of several important surfaces such as catenoids and right cones in $\mathbb{E}^{3}([10])$, and helicoids of the 1st, 2nd and 3rd kind, conjugate of Enneper's surfaces of the 2nd kind and $B$-scrolls in $\mathbb{E}_{1}^{3}$ ([22]) take a somewhat different form; namely,

$$
\Delta G=f(G+C)
$$

for some function $f$ and some constant vector $C$.

A submanifold $M$ is said to be pointwise 1-type Gauss map if its Gauss map satisfies (1.1) for some smooth function $f$ on $M$ and constant vector $C$. A pointwise 1-type Gauss map is called proper if the function $f$ defined by (1.1) is non-constant. A submanifold with pointwise 1-type Gauss map is said to be of the first kind if the vector $C$ in (1.1) is the zero vector. Otherwise, the pointwise 1-type Gauss map is said to be of the second kind.

In [22] the present authors completely classified all possible ruled surfaces with pointwise 1-type Gauss map of the first kind in Minkowski 3 -space $\mathbb{E}_{1}^{3}$ and obtained a new characterization of minimal ruled surfaces. Also, the first author with B.-Y. Chen and M. Choi ([10]) recently investigated surfaces of revolution with pointwise 1-type Gauss map in $\mathbb{E}^{3}$.

Following the ideas of [16], one can also study surfaces in Euclidean 3 -space for which the Gauss map $G$ satisfies a special algebraic condition 
of the form

$$
\Delta G=A G, \quad A \in \mathbb{R}^{3 \times 3} .
$$

Concerning this condition, F. Dillen, J. Pas and L. Verstraelen ([15]) studied surfaces of revolution in Euclidean 3 -space $\mathbb{E}^{3}$ such that its Gauss map $G$ satisfies the condition (1.2). C. Baikoussis and D. E. Blair ([2]) proved that the ruled surfaces in $\mathbb{E}^{3}$ satisfying the condition (1.2) are circular cylinders or planes. C. Baikoussis and L. Verstraelen ([5, 6, 7]) studied the helicoidal surfaces, the translation surfaces and the spiral surfaces in $\mathbb{E}^{3}$ satisfying the condition (1.2). Also, for the Lorentz version, S. M. Choi $([13,14])$ completely classified the surfaces of revolution and the ruled surfaces with non-null base curve satisfying the condition (1.2) in Minkowski 3-space $\mathbb{E}_{1}^{3}$. Furthermore, L. J. Alías, A. Ferrández, P. Lucas and M. A. Meroño ([1]) studied the ruled surfaces with null ruling satisfying the condition (1.2) in Minkowski 3-space $\mathbb{E}_{1}^{3}$. The second named author $([25])$ recently classified translation surfaces satisfying the condition (1.2) in $\mathbb{E}_{1}^{3}$.

Now, we consider the following non-degenerate rotation surfaces in the 4-dimensional pseudo-Euclidean space $\mathbb{E}_{2}^{4}([23])$ :

$$
\begin{aligned}
M_{1}: x(u, v)= & \left(x_{1}(u, v), x_{2}(u, v), x_{3}(u, v), x_{4}(u, v)\right) \\
x_{1}(u, v)= & r(u) \cosh u \sinh v, x_{2}(u, v)=r(u) \sinh u \cosh v \\
x_{3}(u, v)= & r(u) \sinh u \sinh v, x_{4}(u, v)=r(u) \cosh u \cosh v \\
& u, v \in \mathbb{R} .
\end{aligned}
$$

$$
\begin{aligned}
M_{2}: x(u, v)= & \left(x_{1}(u, v), x_{2}(u, v), x_{3}(u, v), x_{4}(u, v)\right) \\
x_{1}(u, v)= & r(u) \sinh u \cos v, x_{2}(u, v)=r(u) \sinh u \sin v \\
x_{3}(u, v)= & r(u) \cosh u \cos v, x_{4}(u, v)=r(u) \cosh u \sin v \\
& u \in \mathbb{R}, v \in[0,2 \pi]
\end{aligned}
$$

where $r(u)$ is a smooth function. We call $M_{1}$ and $M_{2}$ rotation surfaces of type $I$ and $I I$, respectively.

For these surfaces, L. H. Liu ([23]) studied the flat rotation surfaces of finite type in the 4-dimensional pseudo-Euclidean space $\mathbb{E}_{2}^{4}$. On the other hand, the second named author ([24]) completely classified the flat rotation surfaces with finite type Gauss map in the 4-dimensional Euclidean space $\mathbb{E}^{4}$. 
In $\S 2$ we recall some basic formulas and Gauss map of submanifolds in pseudo-Euclidean spaces. In $\S 3$ we study the flat rotation surfaces with finite type Gauss map in the 4-dimensional pseudo-Euclidean space $\mathbb{E}_{2}^{4}$, and in $\S 4$ we investigate the flat rotation surfaces with pointwise 1type Gauss map. In the last two sections we are concerned with the flat rotation surfaces satisfying the partial differential equations $\Delta G=A G$ and $\Delta H=A H$ for some real matrix $A$, where $H$ is the mean curvature vector field of the surfaces.

\section{Preliminaries}

Let $\mathbb{E}_{s}^{m}$ be the $m$-dimensional pseudo-Euclidean space with signature $(s, m-s)$. Then the metric tensor $\tilde{g}$ in $\mathbb{E}_{s}^{m}$ has the form

$$
\tilde{g}=\sum_{i=1}^{m-s}\left(d x_{i}\right)^{2}-\sum_{i=m-s+1}^{m}\left(d x_{i}\right)^{2},
$$

where $\left(x_{1}, x_{2}, \ldots, x_{m}\right)$ is a standard rectangular coordinate system in $\mathbb{E}_{s}^{m}$.

A pseudo-Riemannian sphere and a pseudo-hyperbolic space are very typical examples of nice submanifolds of $\mathbb{E}_{s}^{m}$ defined by respectively as follows : For a point $c \in \mathbb{E}_{s}^{m}$ and $r>0$, we put

$$
\begin{aligned}
& \mathbb{S}_{s}^{m-1}(c, r)=\left\{x \in \mathbb{E}_{s}^{m} \mid\langle x-c, x-c\rangle=r^{2}\right\} \\
& \mathbb{H}_{s-1}^{m-1}(c, r)=\left\{x \in \mathbb{E}_{s}^{m} \mid\langle x-c, x-c\rangle=-r^{2}\right\}
\end{aligned}
$$

where $<, \quad>$ denotes the indefinite inner product on $\mathbb{E}_{s}^{m}$. It is wellknown that $\mathbb{S}_{s}^{m-1}(c, r), \mathbb{H}_{s-1}^{m-1}(c, r)$ are complete pseudo-Riemannian manifolds with constant sectional curvature $r^{-2}$ and $-r^{-2}$, respectively. The pseudo- Riemannian sphere $\mathbb{S}_{s}^{m-1}(c, r)$ is diffeomorphic to $\mathbb{R}^{s} \times$ $\mathbb{S}^{m-1-s}$; the pseudo-hyperbolic space $\mathbb{H}_{s-1}^{m-1}(c, r)$ is diffeomorphic to $\mathbb{S}^{s-1} \times \mathbb{R}^{m-s}$. The hyperbolic space $\mathbb{H}^{m-1}(c, r)$ is defined by

$$
\mathbb{H}^{m-1}(c, r)=\left\{x \in \mathbb{E}_{1}^{m}\left|\langle x-c, x-c\rangle=-r^{2}, x_{m}\right\rangle 0\right\} .
$$

In particular, if $m=2$, the hyperbolic space $\mathbb{H}^{1}(c, r)$ is called the plane hyperbola.

Let $x: M^{n} \longrightarrow \mathbb{E}_{s}^{m}$ be an isometric immersion of an oriented $n$ dimensional pseudo-Riemannian submanifold $M^{n}$ into $\mathbb{E}_{s}^{m}$. From now 
on, a submanifold in $\mathbb{E}_{s}^{m}$ always means pseudo-Riemannian. Let $\bar{\nabla}$ be the Levi-Civita connection of $\mathbb{E}_{s}^{m}$ and $\nabla$ the induced connection on $M^{n}$. Let $e_{1}, \ldots, e_{n}, e_{n+1}, \ldots, e_{m}$ be an adapted local orthonormal frame in $\mathbb{E}_{s}^{m}$ such that $e_{1}, e_{2}, \ldots, e_{n}$ are tangent to $M^{n}$ and $e_{n+1}, e_{n+2}, \ldots, e_{m}$ normal to $M^{n}$. We shall make use of the following convention on the ranges of indices : $1 \leq i, j, \cdots \leq n, \quad n+1 \leq s, t, \cdots \leq m, \quad 1 \leq$ $A, B, \cdots \leq m$.

Let $\omega_{A}$ be the dual 1-form of $e_{A}$ defined by $\omega_{A}(X)=\left\langle e_{A}, X\right\rangle$ and $\varepsilon_{A}=<e_{A}, e_{A}>= \pm 1$. Also, the connection forms $\omega_{A B}$ are defined by

$$
d e_{A}=\sum \varepsilon_{B} \omega_{A B} e_{B}, \quad \omega_{A B}+\omega_{B A}=0 .
$$

Then, the structure equations of $\mathbb{E}_{s}^{m}$ are obtained as follows :

$$
\begin{aligned}
d \omega_{A} & =\sum \varepsilon_{B} \omega_{A B} \wedge \omega_{B}, \\
d \omega_{A B} & =\sum \varepsilon_{C} \omega_{A C} \wedge \omega_{C B} .
\end{aligned}
$$

The canonical forms $\left\{\omega_{A}\right\}$ and the connection forms $\left\{\omega_{A B}\right\}$ restricted to $M^{n}$ are also denoted by the same symbols. We then have

$$
\omega_{s}=0 \text { for } s=n+1, \ldots, m \text {. }
$$

It follows from (2.4) and the Cartan lemma that the exterior derivative of (2.4) gives rise to

$$
\omega_{i s}=\sum_{j} \varepsilon_{j} h_{i j}^{s} \omega_{j}, \quad h_{i j}^{s}=h_{j i}^{s} .
$$

The mean curvature vector $H$ of $M^{n}$ in $\mathbb{E}_{s}^{m}$ is defined by

$$
H=\frac{1}{n} \sum_{s=n+1}^{m} \sum_{i=1}^{n} \varepsilon_{i} \varepsilon_{s} h_{i i}^{s} e_{s} .
$$

For lifting or lowering indices we have

$$
\omega^{A}=\varepsilon_{A} \omega_{A} \quad \text { and } \quad \omega_{A}^{B}=\varepsilon_{B} \omega_{A B} .
$$

Furthermore, we have the covariant differentiation on $e_{i}$ given by

$$
d e_{i}=\sum_{A} \varepsilon_{A} \omega_{i A} e_{A} \quad \text { or } \quad \bar{\nabla}_{i} e_{j}=\sum_{B} \varepsilon_{B} \omega_{j B}\left(e_{i}\right) e_{B}
$$


For any real valued function $f$ on $M^{n}$ the Laplacian $\Delta f$ of $f$ is defined by

$$
\Delta f=-\sum_{i} \varepsilon_{i}\left(\bar{\nabla} e_{i} \bar{\nabla} e_{i} f-\bar{\nabla}_{\nabla_{i} e_{i}} f\right)
$$

Let us now define the Gauss map $G$ of a submanifold $M^{n}$ into $G(n, m)$ in $\bigwedge^{n} \mathbb{E}_{s}^{m}$, where $G(n, m)$ is the Grassmannian manifold consisting of all oriented $n$-planes through the origin of $\mathbb{E}_{s}^{m}$ and $\bigwedge^{n} \mathbb{E}_{s}^{m}$ is the vector space obtained by the exterior product of $n$ vectors in $\mathbb{E}_{s}^{m}$. Let $e_{i_{1}} \wedge \cdots \wedge e_{i_{n}}$ and $f_{j_{1}} \wedge \cdots \wedge f_{j_{n}}$ be two vectors of $\wedge^{n} \mathbb{E}_{s}^{m}$, where $\left\{e_{1}, e_{2}, \ldots, e_{m}\right\}$ and $\left\{f_{1}, f_{2}, \ldots, f_{m}\right\}$ are the orthonormal bases of $\mathbb{E}_{s}^{m}$. Define an indefinite inner product $\langle$,$\rangle on \bigwedge^{n} \mathbb{E}_{s}^{m}$ by

$$
\left\langle e_{i_{1}} \wedge \cdots \wedge e_{i_{n}}, f_{j_{1}} \wedge \cdots \wedge f_{j_{n}}\right\rangle=\operatorname{det}\left(\left\langle e_{i_{l}}, f_{j_{k}}\right\rangle\right) .
$$

Therefore, for some positive integer $k$, we may identify $\bigwedge^{n} \mathbb{E}_{s}^{m}$ with some pseudo-Euclidean space $\mathbb{E}_{k}^{N}$, where $N=\left(\begin{array}{l}m \\ n\end{array}\right)$. The map $G: M^{n} \longrightarrow$ $G(n, m) \subset \mathbb{E}_{k}^{N}$ defined by $G(p)=\left(e_{1} \wedge e_{2} \wedge \cdots \wedge e_{n}\right)(p)$ is called the Gauss map of $M^{n}$ that is a smooth map which carries a point $p$ in $M^{n}$ into the oriented $n$-plane in $\mathbb{E}_{s}^{m}$ obtained from the parallel translation of the tangent space of $M^{n}$ at $p$ in $\mathbb{E}_{s}^{m}$. Then, the Gauss image $G(M)$ can be viewed as $G(M) \subset \mathbb{S}_{k}^{N-1}(\mathbf{0}, 1)$.

\section{Rotation surfaces with finite type Gauss map}

In this section we study the flat rotation surfaces of type $I$ or type $I I$ with finite type Gauss map in the 4-dimensional pseudo-Euclidean space $\mathbb{E}_{2}^{4}$.

Let $M$ be the rotation surface of type $I$ (resp. type $I I$ ) in $\mathbb{E}_{2}^{4}$ defined by $(1.3 \mathrm{a})$ (resp. $(1.3 \mathrm{~b})$ ). We choose a moving frame $e_{1}, e_{2}, e_{3}, e_{4}$ such that $e_{1}, e_{2}$ are tangent to $M$ of type $I$ and $e_{3}, e_{4}$ are normal to $M$ of type $I$ and choose a moving frame $\bar{e}_{1}, \bar{e}_{2}, \bar{e}_{3}, \bar{e}_{4}$ such that $\bar{e}_{1}, \bar{e}_{2}$ are tangent to $M$ of type $I I$ and $\bar{e}_{3}, \bar{e}_{4}$ are normal to $M$ of type $I I$ which are given by the following ([23]) :

$$
\left\{\begin{array}{l}
e_{1}=(\cosh u \cosh v, \sinh u \sinh v, \sinh u \cosh v, \cosh u \sinh v) \\
e_{2}=\frac{1}{A_{1}}\left(A_{2} \sinh v, A_{3} \cosh v, A_{3} \sinh v, A_{2} \cosh v\right) \\
e_{3}=\frac{1}{A_{1}}\left(A_{3} \sinh v, A_{2} \sinh v, A_{2} \sinh v, A_{3} \cosh v\right) \\
e_{4}=-\varepsilon(\sinh u \cosh v, \cosh u \sinh v, \cosh u \cosh v, \sinh u \sinh v),
\end{array}\right.
$$


$(3.1 \mathrm{~b})$

$$
\left\{\begin{array}{l}
\bar{e}_{1}=(-\sinh u \sin v, \sinh u \cos v,-\cosh u \sin v, \cosh u \cos v) \\
\bar{e}_{2}=\frac{1}{A_{1}}\left(A_{3} \cos v, A_{3} \sin v, A_{2} \cos v, A_{2} \sin v\right) \\
\bar{e}_{3}=\frac{1}{A_{1}}\left(A_{2} \cos v, A_{2} \sin v, A_{3} \cos v, A_{3} \sin v\right) \\
\bar{e}_{4}=\varepsilon(\cosh u \sin v,-\cosh u \cos v, \sinh u \sin v,-\sinh u \cos v),
\end{array}\right.
$$

where we put $A_{1}=\sqrt{\varepsilon\left(r^{2}-r^{\prime 2}\right)}, \varepsilon= \pm 1, \varepsilon\left(r^{2}-r^{\prime 2}\right)>0, A_{2}=r^{\prime} \cosh u+$ $r \sinh u, A_{3}=r^{\prime} \sinh u+r \cosh u$. Then, it is easily seen that

$$
\begin{aligned}
& <e_{1}, e_{1}>=-<e_{4}, e_{4}>=1, \quad<e_{2}, e_{2}>=-<e_{3}, e_{3}>=\varepsilon \\
& -<\bar{e}_{1}, \bar{e}_{1}>=<\bar{e}_{4}, \bar{e}_{4}>=1, \quad<\bar{e}_{2}, \bar{e}_{2}>=-<\bar{e}_{3}, \bar{e}_{3}>=\varepsilon .
\end{aligned}
$$

Also, we have

$$
\begin{aligned}
& \omega_{1}=r d v, \quad \omega_{2}=\varepsilon \sqrt{\varepsilon\left(r^{2}-r^{\prime 2}\right)} d u=\varepsilon A_{1} d u \\
& \bar{\omega}_{1}=-r d v, \quad \bar{\omega}_{2}=\varepsilon A_{1} d u,
\end{aligned}
$$

which imply $e_{1}, e_{2}, \bar{e}_{1}$ and $\bar{e}_{2}$ are written as

$$
\begin{aligned}
& e_{1}=\frac{1}{r} \frac{\partial}{\partial v}, \quad e_{2}=\frac{1}{\varepsilon A_{1}} \frac{\partial}{\partial u} \\
& \bar{e}_{1}=-\frac{1}{r} \frac{\partial}{\partial v}, \quad \bar{e}_{2}=\frac{1}{\varepsilon A_{1}} \frac{\partial}{\partial u} .
\end{aligned}
$$

As is introduced in section 2, the Gauss map $G$ (resp. $\bar{G}$ ) of $M$ of type $I$ (resp. type $I I$ ) is given by $G=e_{1} \wedge e_{2}$ (resp. $\left.\bar{G}=\vec{e}_{1} \wedge \vec{e}_{2}\right)$. Using (2.3), (2.4), (3.1 a), (3.1 b), (3.2 a) and (3.2 b) we can obtain the coefficients of the second fundamental form $h$ and the connection forms $\omega_{A B}$ as follows:

$$
\left\{\begin{array}{l}
h_{11}^{3}=-\alpha, \quad h_{12}^{3}=h_{21}^{3}=0, \quad h_{22}^{3}=\varepsilon \beta \\
h_{11}^{4}=h_{22}^{4}=0, \quad h_{12}^{4}=h_{21}^{4}=\varepsilon \alpha
\end{array}\right.
$$

$$
\left\{\begin{array}{l}
\bar{h}_{11}^{3}=\alpha, \quad \bar{h}_{12}^{3}=\bar{h}_{21}^{3}=0, \quad \bar{h}_{22}^{3}=\varepsilon \beta \\
\bar{h}_{11}^{4}=\bar{h}_{22}^{4}=0, \quad \bar{h}_{12}^{4}=\bar{h}_{21}^{4}=-\varepsilon \alpha
\end{array}\right.
$$

$$
\left\{\begin{array}{l}
\omega_{12}=-\alpha \kappa \omega_{1}, \quad \omega_{13}=-\alpha \omega_{1}, \quad \omega_{14}=\alpha \omega_{2} \\
\omega_{23}=\beta \omega_{2}, \quad \omega_{24}=\varepsilon \alpha \omega_{1}, \quad \omega_{34}=\varepsilon \alpha \kappa \omega_{1}
\end{array}\right.
$$




$$
\left\{\begin{array}{l}
\bar{\omega}_{12}=-\alpha \kappa \bar{\omega}_{1}, \quad \bar{\omega}_{13}=-\alpha \bar{\omega}_{1}, \quad \bar{\omega}_{14}=-\alpha \bar{\omega}_{2}, \\
\bar{\omega}_{23}=\beta \bar{\omega}_{2}, \quad \bar{\omega}_{24}=\varepsilon \alpha \bar{\omega}_{1}, \quad \bar{\omega}_{34}=\varepsilon \alpha \kappa \bar{\omega}_{1},
\end{array}\right.
$$

where we have put $\alpha=A_{1}^{-1}, \quad \kappa=\frac{r^{\prime}}{r}, \quad \beta=\varepsilon A_{1}^{-3}\left(2 r^{\prime 2}-r r^{\prime \prime}-r^{2}\right)$. The Gaussian curvature $K$ of $M$ of type $I$ and $\bar{K}$ that of $M$ of type $I I$ are respectively given by

$$
K=\sum_{s=3}^{4} \varepsilon_{s}\left(h_{11}^{s} h_{22}^{s}-h_{12}^{s} h_{21}^{s}\right)=\varepsilon A_{1}^{-4}\left(r^{\prime 2}-r r^{\prime \prime}\right)
$$

and

$$
\bar{K}=\varepsilon A_{1}^{-4}\left(r r^{\prime \prime}-r^{2}\right) .
$$

Moreover, combining (2.7), (3.4 a), (3.4 b), (3.5 a) and (3.5 b) we have

$$
\left\{\begin{array}{l}
\bar{\nabla}_{e_{1}} e_{1}=-\varepsilon \alpha \kappa e_{2}+\varepsilon \alpha e_{3}, \quad \bar{\nabla}_{e_{1}} e_{2}=\alpha \kappa e_{1}-\varepsilon \alpha e_{4}, \\
\bar{\nabla}_{e_{1}} e_{3}=\alpha e_{1}-\varepsilon \alpha \kappa e_{4}, \quad \bar{\nabla}_{e_{1}} e_{4}=-\alpha e_{2}+\alpha \kappa e_{3}, \\
\bar{\nabla} e_{2} e_{1}=-\alpha e_{4}, \quad \bar{\nabla} e_{2} e_{2}=-\varepsilon \beta e_{3}, \\
\bar{\nabla} e_{2} e_{3}=-\varepsilon \beta e_{2}, \quad \bar{\nabla} e_{2} e_{4}=-\alpha e_{1}, \\
\nabla e_{1} e_{1}=-\varepsilon \alpha \kappa e_{2}, \quad \nabla_{e_{2}} e_{2}=0
\end{array}\right.
$$

and

$$
\left\{\begin{array}{l}
\bar{\nabla} \bar{e}_{1} \bar{e}_{1}=-\varepsilon \alpha \kappa \bar{e}_{2}+\varepsilon \alpha \bar{e}_{3}, \quad \bar{\nabla} \bar{e}_{1} \bar{e}_{2}=-\alpha \kappa \bar{e}_{1}+\varepsilon \alpha \bar{e}_{4}, \\
\bar{\nabla} \bar{e}_{1} \bar{e}_{3}=-\alpha \bar{e}_{1}+\varepsilon \alpha \kappa \bar{e}_{4}, \quad \bar{\nabla} \bar{e}_{1} \bar{e}_{4}=-\alpha \bar{e}_{2}+\alpha \kappa \bar{e}_{3}, \\
\bar{\nabla}_{\bar{e}_{2}} \bar{e}_{1}=-\alpha \bar{e}_{4}, \quad \bar{\nabla} \bar{e}_{2} \bar{e}_{2}=-\varepsilon \beta \bar{e}_{3}, \\
\bar{\nabla} \bar{e}_{2} \bar{e}_{3}=-\varepsilon \beta \bar{e}_{2}, \quad \quad \bar{\nabla} \bar{e}_{2} \bar{e}_{4}=-\alpha \bar{e}_{1}, \\
\nabla \bar{e}_{1} \bar{e}_{1}=-\varepsilon \alpha \kappa \bar{e}_{2}, \quad \nabla \bar{e}_{2} \bar{e}_{2}=0 .
\end{array}\right.
$$

Suppose that the rotation surface $M$ of type $I$ or $I I$ is flat. Then, (3.6 a) and (3.6 b) imply $r r^{\prime \prime}=r^{2}$. Thus $r(u)=c e^{k u}$ for some constants $c \neq 0$ and $k$. If necessary, by an appropriate homothetic transformation we may assume that $c=1$. Thus we have

$$
r(u)=e^{k u}, \quad \alpha=-\beta=\frac{e^{-k u}}{\sqrt{\varepsilon\left(1-k^{2}\right)}}, \quad \varepsilon\left(1-k^{2}\right)>0 .
$$

From $(3.3 \mathrm{a}),(3.3 \mathrm{~b})$ and $(3.8)$ we get

$$
e_{1}(\alpha)=\bar{e}_{1}(\alpha)=0, \quad e_{2}(\alpha)=\bar{e}_{2}(\alpha)=-\varepsilon k \alpha^{2}, \quad \kappa=k=\text { constant }
$$


If $k=0$, then $r(u)=1$. In this case, the surface $M$ of type $I$ is the product of two plane hyperbolas, and the surface $M$ of type $I I$ is the product of a plane circle and a plane hyperbola.

Now, we assume that $k \neq 0$. By using (2.8), (3.7 a), (3.7 b), (3.8), (3.9) and straight-forward computation, the Laplacians $\Delta G$ and $\Delta \bar{G}$ of the Gauss maps $G$ and $\bar{G}$ can be expressed as

$$
\text { a) } \Delta G=-4 \varepsilon \alpha^{2} e_{1} \wedge e_{2}+(3 \varepsilon k-k) \alpha^{2} e_{1} \wedge e_{3}-(\varepsilon k+k) \alpha^{2} e_{2} \wedge e_{4}
$$

and

$$
\text { b) } \Delta \bar{G}=-4 \varepsilon \alpha^{2} \bar{e}_{1} \wedge \bar{e}_{2}+(3 \varepsilon k+k) \alpha^{2} \bar{e}_{1} \wedge \bar{e}_{3}+(\varepsilon k-k) \alpha^{2} \bar{e}_{2} \wedge \bar{e}_{4}
$$

On the other hand, by a direct calculation we have

$$
\left\{\begin{aligned}
& \Delta\left(\alpha^{n}\right)=k^{2}(n-\varepsilon(n+1) n) \alpha^{n+2} \\
& \Delta\left(e_{1} \wedge e_{3}\right)=-(k+\varepsilon k) \alpha^{2} e_{1} \wedge e_{2}+\left(2 \varepsilon k^{2}-2 \varepsilon\right) \alpha^{2} e_{1} \wedge e_{3} \\
&-\left(2 k^{2}+2\right) \alpha^{2} e_{2} \wedge e_{4}+(3 k-\varepsilon k) \alpha^{2} e_{3} \wedge e_{4} \\
& \Delta\left(e_{2} \wedge e_{4}\right)=(3 k-\varepsilon k) \alpha^{2} e_{1} \wedge e_{2}-\left(2 k^{2}+2\right) \alpha^{2} e_{1} \wedge e_{3} \\
&+\left(2 \varepsilon k^{2}-2 \varepsilon\right) \alpha^{2} e_{2} \wedge e_{4}-(k+\varepsilon k) \alpha^{2} e_{3} \wedge e_{4} \\
& \Delta\left(e_{3} \wedge e_{4}\right)=-(\varepsilon k+k) \alpha^{2} e_{1} \wedge e_{3}+(3 \varepsilon k-k) \alpha^{2} e_{2} \wedge e_{4} \\
&-4 \varepsilon \alpha^{2} e_{3} \wedge e_{4}
\end{aligned}\right.
$$

and

$$
\left\{\begin{aligned}
& \Delta\left(\alpha^{n}\right)=-k^{2}(n+\varepsilon(n+1) n) \alpha^{n+2} \\
& \Delta\left(\bar{e}_{1} \wedge \bar{e}_{3}\right)=(k-\varepsilon k) \alpha^{2} \bar{e}_{1} \wedge \bar{e}_{2}+\left(2 \varepsilon k^{2}-2 \varepsilon\right) \alpha^{2} \bar{e}_{1} \wedge \bar{e}_{3} \\
&-\left(2 k^{2}+2\right) \alpha^{2} \bar{e}_{2} \wedge \bar{e}_{4}+(3 k+\varepsilon k) \alpha^{2} \bar{e}_{3} \wedge \bar{e}_{4}, \\
& \Delta\left(\bar{e}_{2} \wedge \bar{e}_{4}\right)=(3 k+\varepsilon k) \alpha^{2} \bar{e}_{1} \wedge \bar{e}_{2}-\left(2 k^{2}+2\right) \alpha^{2} \bar{e}_{1} \wedge \bar{e}_{3} \\
&+\left(2 \varepsilon k^{2}-2 \varepsilon\right) \alpha^{2} \bar{e}_{2} \wedge \bar{e}_{4}+(k-\varepsilon k) \alpha^{2} \bar{e}_{3} \wedge \bar{e}_{4}, \\
& \Delta\left(\bar{e}_{3} \wedge \bar{e}_{4}\right)=(\varepsilon k-k) \alpha^{2} \bar{e}_{1} \wedge \bar{e}_{3}+(3 \varepsilon k+k) \alpha^{2} \bar{e}_{2} \wedge \bar{e}_{4}-4 \varepsilon \alpha^{2} \bar{e}_{3} \wedge \bar{e}_{4} .
\end{aligned}\right.
$$

Therefore, from (2.8), (3.10 a), (3.10 b), (3.11 a) and (3.11 b) we obtain

$$
\Delta^{2} G
$$

$$
\begin{aligned}
= & \left(-4 \varepsilon k^{2}+12 k^{2}+16\right) \alpha^{4} e_{1} \wedge e_{2} \\
& +\left(12 \varepsilon k^{3}-12 k^{3}-8 \varepsilon k-16 k\right) \alpha^{4} e_{1} \wedge e_{3} \\
& +\left(-4 \varepsilon k^{3}+4 k^{3}-8 k\right) \alpha^{4} e_{2} \wedge e_{4}+\left(4 \varepsilon k^{2}+4 k^{2}\right) \alpha^{4} e_{3} \wedge e_{4}
\end{aligned}
$$


and

$(3.12 \mathrm{~b})$

$$
\begin{aligned}
\Delta^{2} \bar{G}= & \left(20 \varepsilon k^{2}+28 k^{2}+16\right) \alpha^{4} \bar{e}_{1} \wedge \bar{e}_{2} \\
& +\left(-12 \varepsilon k^{3}-12 k^{3}-24 \varepsilon k-16 k\right) \alpha^{4} \bar{e}_{1} \wedge \bar{e}_{3} \\
& +\left(-4 \varepsilon k^{3}-4 k^{3}-24 k\right) \alpha^{4} \bar{e}_{2} \wedge \bar{e}_{4}+\left(20 \varepsilon k^{2}+12 k^{2}\right) \alpha^{4} \bar{e}_{3} \wedge \bar{e}_{4} .
\end{aligned}
$$

Proceeding by induction we can have

$$
\text { a) } \Delta^{n} G=a_{n} \alpha^{2 n} e_{1} \wedge e_{2}+b_{n} \alpha^{2 n} e_{1} \wedge e_{3}+c_{n} \alpha^{2 n} e_{2} \wedge e_{4}+d_{n} \alpha^{2 n} e_{3} \wedge e_{4}
$$

and

(3.13 b) $\Delta^{n} \bar{G}=\bar{a}_{n} \alpha^{2 n} \bar{e}_{1} \wedge \bar{e}_{2}+\bar{b}_{n} \alpha^{2 n} \bar{e}_{1} \wedge \bar{e}_{3}+\bar{c}_{n} \alpha^{2 n} \bar{e}_{2} \wedge \bar{e}_{4}+\bar{d}_{n} \alpha^{2 n} \bar{e}_{3} \wedge \bar{e}_{4}$, where $d_{1}=0, \bar{d}_{1}=0$ and $a_{n}, b_{n}, c_{n}, d_{n}, \bar{a}_{n}, \bar{b}_{n}, \bar{c}_{n}, \bar{d}_{n}$ are some constants for $n \geq 1$. In fact, $(3.10 \mathrm{a}),(3.10 \mathrm{~b}),(3.12 \mathrm{a})$ and $(3.12 \mathrm{~b})$ show that $(3.13 \mathrm{a})$ and $(3.13 \mathrm{~b})$ are true for $n=1,2$. Suppose that $(3.13 \mathrm{a})$ and $(3.13 \mathrm{~b})$ are true for $m$. Then, by using (3.11 a), (3.11 b), (3.13 a) and (3.13 b) one can check after a long computation

$$
\begin{aligned}
& \Delta^{m+1} G \\
= & \Delta\left(\alpha^{2 m}\right)\left(a_{m} e_{1} \wedge e_{2}+b_{m} e_{1} \wedge e_{3}+c_{m} e_{2} \wedge e_{4}+d_{m} e_{3} \wedge e_{4}\right) \\
& +\alpha^{2 m} \Delta\left(a_{m} e_{1} \wedge e_{2}+b_{m} e_{1} \wedge e_{3}+c_{m} e_{2} \wedge e_{4}+d_{m} e_{3} \wedge e_{4}\right) \\
& -2 e_{2}\left(\alpha^{2 m}\right) \bar{\nabla}_{e_{2}}\left(a_{m} e_{1} \wedge e_{2}+b_{m} e_{1} \wedge e_{3}+c_{m} e_{2} \wedge e_{4}+d_{m} e_{3} \wedge e_{4}\right) \\
= & a_{m+1} \alpha^{2 m+2} e_{1} \wedge e_{2}+b_{m+1} \alpha^{2 m+2} e_{1} \wedge e_{3}+c_{m+1} \alpha^{2 m+2} e_{2} \wedge e_{4} \\
& +d_{m+1} \alpha^{2 m+2} e_{3} \wedge e_{4}
\end{aligned}
$$

and

$$
\begin{aligned}
\Delta^{m+1} \bar{G}= & \overline{a_{m+1}} \alpha^{2 m+2} \bar{e}_{1} \wedge \bar{e}_{2}+\overline{b_{m+1}} \alpha^{2 m+2} \bar{e}_{1} \wedge \bar{e}_{3}+\overline{c_{m+1}} \alpha^{2 m+2} \bar{e}_{2} \wedge \bar{e}_{4} \\
& +\overline{d_{m+1}} \alpha^{2 m+2} \bar{e}_{3} \wedge \bar{e}_{4},
\end{aligned}
$$

where we have put

$$
\left\{\begin{aligned}
a_{m+1}= & \left(2 m k^{2}-2 \varepsilon m k^{2}(2 m+1)-4 \varepsilon\right) a_{m}+(4 m k-k-\varepsilon k) b_{m} \\
& +(4 \varepsilon m k+3 k-\varepsilon k) c_{m} \\
b_{m+1}= & (4 m k-3 \varepsilon k-k) a_{m}+\left(2 m k^{2}-2 \varepsilon m k^{2}(2 m+1)+2 \varepsilon k^{2}\right. \\
& -2 \varepsilon) b_{m}-\left(2 k^{2}+2\right) c_{m}+(4 \varepsilon m k-\varepsilon k-k) d_{m} \\
c_{m+1}= & (4 \varepsilon m k-\varepsilon k-k) a_{m}-\left(2 k^{2}+2\right) b_{m}+\left(2 m k^{2}\right. \\
& \left.-2 \varepsilon m k^{2}(2 m+1)+2 \varepsilon k^{2}-2 \varepsilon\right) c_{m}+(4 m k+3 \varepsilon k-k) d_{m} \\
d_{m+1}= & (4 \varepsilon m k+3 k-\varepsilon k) b_{m}+(4 m k-k-\varepsilon k) c_{m} \\
& +\left(2 m k^{2}-2 \varepsilon m k^{2}(2 m+1)-4 \varepsilon\right) d_{m}
\end{aligned}\right.
$$


and

$$
\left\{\begin{aligned}
& \overline{a_{m+1}}=\left(-2 m k^{2}-2 \varepsilon m k^{2}(2 m+1)-4 \varepsilon\right) \bar{a}_{m}+(4 m k+k-\varepsilon k) \bar{b}_{m} \\
&+(4 \varepsilon m k+3 k+\varepsilon k) \bar{c}_{m} \\
& \overline{b_{m+1}}=(4 m k+3 \varepsilon k+k) \bar{a}_{m}+\left(-2 m k^{2}-2 \varepsilon m k^{2}(2 m+1)\right. \\
&\left.+2 \varepsilon k^{2}-2 \varepsilon\right) \bar{b}_{m}-\left(2 k^{2}+2\right) \bar{c}_{m}+(4 \varepsilon m k+\varepsilon k-k) \bar{d}_{m} \\
& \overline{c_{m+1}}=(4 \varepsilon m k+\varepsilon k-k) \bar{a}_{m}-\left(2 k^{2}+2\right) \bar{b}_{m} \\
& \\
&+\left(-2 m k^{2}-2 \varepsilon m k^{2}(2 m+1)+2 \varepsilon k^{2}-2 \varepsilon\right) \bar{c}_{m} \\
&+(4 m k+3 \varepsilon k+k) \bar{d}_{m} \\
& \overline{d_{m+1}}=4 \varepsilon m k+3 k+\varepsilon k) \bar{b}_{m}+(4 m k+k-\varepsilon k) \bar{c}_{m} \\
&+\left(-2 m k^{2}-2 \varepsilon m k^{2}(2 m+1)-4 \varepsilon\right) \bar{d}_{m}
\end{aligned}\right.
$$

Therefore, we can conclude that (3.13 a) and (3.13 b) hold for any positive integer $n$.

We suppose that the flat rotation surface $M$ of type $I$ has finite type Gauss map in $\mathbb{E}_{2}^{4}$. Then, there exist real numbers $l_{1}, l_{2}, \ldots, l_{k}$ such that

$$
\Delta^{k+1} G+l_{k} \Delta^{k} G+\cdots+l_{1} \Delta G=0
$$

for some positive integer $k$. By using (3.13 a), (3.14) is nothing but

$$
P(\alpha) e_{1} \wedge e_{2}+Q(\alpha) e_{1} \wedge e_{3}+R(\alpha) e_{2} \wedge e_{4}+S(\alpha) e_{3} \wedge e_{4}=0
$$

where $P(s), Q(s), R(s)$ and $S(s)$ are some polynomials in $s$ of degree $2 k+2$ with constant coefficients. It is obvious that $P(\alpha)=0, Q(\alpha)=$ $0, R(\alpha)=0$ and $S(\alpha)=0$ because $e_{i} \wedge e_{j}(i \neq j)$ are linearly independent. Thus, $\alpha$ must be a constant.

Next, we assume that the flat rotation surface $M$ of type $I I$ is of finite type Gauss map in $\mathbb{E}_{2}^{4}$. Then, by the similar discussion of that of $M$ of type $I$ we can see that $\alpha$ is also a constant. Therefore, from (3.8) we have $k=0$ and thus $r(u)=1$ and $\alpha=-\beta=1$. Also, from $(3.10 \mathrm{a})$ (resp. $(3.10 \mathrm{~b})$ ) we obtain $\Delta G=-4 \varepsilon G$ (resp. $\Delta \bar{G}=-4 \varepsilon \bar{G})$, that is, the surface $M$ of type $I$ (resp. type $I I$ ) is of 1-type Gauss map. Consequently, from (1.3 a) the surface $M$ of type $I$ is the product of two plane hyperbolas, and from $(1.3 \mathrm{~b})$ the surface $M$ of type $I I$ is the product of a plane circle and a plane hyperbola.

Thus we have 
THeorem 3.1. Let $M$ be a flat rotation surface of type I (resp. type II) in $\mathbb{E}_{2}^{4}$. Then, the following are equivalent:

1. the Gauss map $G$ on $M$ is of finite type,

2. the Gauss map $G$ on $M$ is of 1-type,

3. $M$ is the product of two plane hyperbolas (resp. the product of a plane circle and a plane hyperbola).

\section{Rotation surfaces with pointwise 1-type Gauss map}

In this section we investigate the flat rotation surfaces of type $I$ (resp. type $I I$ ) in $\mathbb{E}_{2}^{4}$ with pointwise 1-type Gauss map satisfying (1.1). If $M$ is the flat rotation surface of type $I$, then we have from (1.1)

$$
\Delta G=f(G+C)
$$

for some function $f$ and some constant vector $C$.

We may assume that the rotation surface $M$ of type $I$ is parametrized by (1.3 a). From (2.9), (3.10 a) and (4.1) we have

$$
\begin{aligned}
& -4 \alpha^{2}=\varepsilon f+f<C, e_{1} \wedge e_{2}>, \\
& (\varepsilon k-3 k) \alpha^{2}=f<C, e_{1} \wedge e_{3}>, \\
& (k+\varepsilon k) \alpha^{2}=f<C, e_{2} \wedge e_{4}>.
\end{aligned}
$$

We then note that a smooth function $f$ is non-zero. In fact, if $f$ vanishes on some open subset $\mathcal{U}$, then by (4.1) and (3.10 a) $\alpha$ is zero on $\mathcal{U}$, which is a contradiction. Let $\mathcal{W}$ be the open subset of $M$ consisting of points where $f$ is non-zero. We now consider the matters on $\mathcal{W}$ for a while. Then, we obtain from (4.1)

$$
<C, e_{1} \wedge e_{4}>=0,<C, e_{2} \wedge e_{3}>=0,<C, e_{3} \wedge e_{4}>=0 .
$$

By differentiating (4.5) with respect to $u$ and using the first and third equation in (4.5), we get

$$
k<C, e_{1} \wedge e_{3}>-<C, e_{1} \wedge e_{2}>-\varepsilon k<C, e_{2} \wedge e_{4}>=0
$$

and

$$
\varepsilon<C, e_{2} \wedge e_{4}>+<C, e_{1} \wedge e_{3}>=0,
$$


which imply

$$
<C, e_{1} \wedge e_{2}>+2 \varepsilon k<C, e_{2} \wedge e_{4}>=0 .
$$

Combining (4.3), (4.4) and (4.7) we then have

$$
\varepsilon k-k=0 .
$$

If $\varepsilon=-1$, then we have $k=0$. Together with (3.8), we have $r(u)=1$ and $\alpha(u)=1$. So, the function $f$ is a constant and $C=0$. Therefore, the Gauss map $G$ is of global 1-type, that is, $\Delta G=4 G$ because of (3.10 a). Consequently, from Theorem 3.1 the surface $M$ of type $I$ is the product of two plane hyperbolas.

If $\varepsilon=1$, then we have from $(4.2),(4.4)$ and $(4.8)$

$$
f=4 k^{2} \alpha^{2}-4 \alpha^{2}
$$

Thus, a smooth function $f$ depends only on $u$.

On the other hand, by (3.3 a), (3.7 a) and (4.4) we get

$$
2 k \alpha^{2} f^{\prime}=\left(4 k \alpha \alpha^{\prime}+4 \alpha^{2}+f\right) f
$$

where the prime denotes the differentiation with respect to $u$. From (4.10) and (4.11) we thus have

$$
k^{2}\left(1-k^{2}\right)=0
$$

which gives $k=0$ because of (3.8). Hence, $r(u)=1$ and $\alpha(u)=1$. In this case we can also show that a smooth function $f$ is a constant and $C=0$. Therefore the Gauss map is of 1-type by (3.10 a), that is, $\Delta G=-4 G$. By Theorem 3.1 the surface $M$ of type $I$ is the product of two plane hyperbolas.

Similarly, we can prove that the flat rotation surface $M$ of type $I I$ with pointwise 1-type Gauss map has global 1-type Gauss map. Consequently, from Theorem 3.1 the surface $M$ of type $I I$ is the product of a plane circle and a plane hyperbola.

In conclusion, we obtain

THEOREM 4.1. A flat rotation surface $M$ of type $I$ or $I I$ with pointwise 1-type Gauss map in $\mathbb{E}_{2}^{4}$ has global 1-type Gauss map, i.e., $M$ is either the product of two plane hyperbolas or the product of a plane circle and a plane hyperbola. 


\section{Rotation surfaces satisfying $\Delta G=A G$}

In this section we study the flat rotation surfaces of type $I$ or type $I I$ in $\mathbb{E}_{2}^{4}$ satisfying the condition (1.2). For a while we only consider the flat rotation surface $M$ is of type $I$. Then, we have

$$
\Delta G=A G, \quad A \in \mathbb{R}^{6 \times 6} .
$$

We may assume that $M$ is parametrized by (1.3 a). From (5.1) and $(3.10$ a) we have

$$
A\left(e_{1} \wedge e_{2}\right)=-4 \varepsilon \alpha^{2} e_{1} \wedge e_{2}+(3 \varepsilon k-k) \alpha^{2} e_{1} \wedge e_{3}-(\varepsilon k+k) \alpha^{2} e_{2} \wedge e_{4}
$$

Since $\alpha$ is a non-zero function, by (3.7 a), (3.9) and (5.2) we obtain

$$
A\left(e_{2} \wedge e_{3}\right)+A\left(e_{1} \wedge e_{4}\right)=\left(4 \varepsilon k^{2}-4 \varepsilon\right) \alpha^{2} e_{1} \wedge e_{4}+\left(4 \varepsilon k^{2}-4 \varepsilon\right) \alpha^{2} e_{2} \wedge e_{3} .
$$

On the other hand, the equation ( 3.7 a) gives

$$
\bar{\nabla} e_{2} A\left(e_{2} \wedge e_{3}\right)=0 \text { and } \bar{\nabla} e_{2} A\left(e_{1} \wedge e_{4}\right)=0,
$$

which imply

$$
\varepsilon\left(k^{2}-1\right) \alpha^{2} \alpha^{\prime}=0
$$

with the aid of (3.3 a), (3.7 a) and (5.3). Thus, $\alpha$ must be a constant because of (3.8). By (3.8) we have $k=0$ and thus $r(u)=1$ and $\alpha=$ $-\beta=1$. Consequently, from (3.10 a) the surface $M$ of type $I$ has 1-type Gauss map.

Similarly, we can prove that the flat rotation surface $M$ of type $I I$ satisfying the condition (5.1) has 1-type Gauss map.

Thus we have

TheOREM 5.1. Let $M$ be a flat rotation surface of type $I$ or $I I$ in $\mathbb{E}_{2}^{4}$. Then, $M$ has global 1-type Gauss map $G$ if and only if the Gauss map $G$ satisfies a partial differential equation

$$
\Delta G=A G, \quad A \in \mathbb{R}^{6 \times 6} .
$$




\section{Rotation surfaces satisfying $\Delta H=A H$}

In this section we look into the flat rotation surfaces of type $I$ (resp. type $I I)$ in $\mathbb{E}_{2}^{4}$ satisfying the following condition

$$
\Delta H=A H, \quad(\text { resp. } \quad \Delta \bar{H}=\bar{A} \bar{H}) \quad A, \bar{A} \in \mathbb{R}^{4 \times 4},
$$

where $H$ (resp. $\bar{H}$ ) denotes the mean curvature vector on $M$ of type $I$ (resp. type $I I$ ).

Let $M$ be a rotation surface of the type $I$ or $I I$. We assume that the rotation surface $M$ is flat. Then, from (2.6), $(3.4 \mathrm{a}),(3.4 \mathrm{~b})$ and (3.8) the mean curvature vector $H$ of $M$ of type $I$ and $\bar{H}$ that of $M$ of type $I I$ are respectively given by

$$
H=\frac{1}{2} \sum_{s=3}^{4} \sum_{i=1}^{2} \varepsilon_{i} \varepsilon_{s} h_{i i}^{s} e_{s}=\varepsilon \alpha e_{3}
$$

and

$$
\bar{H}=\varepsilon \alpha \bar{e}_{3} .
$$

By straightforward computation, the Laplacians $\Delta H$ and $\Delta \vec{H}$ of the mean curvature vectors $H$ and $\bar{H}$ with the help of (2.8), (6.2 a) and (6.2 b) turn out to be

$$
\Delta H=(3 k-\varepsilon k) \alpha^{3} e_{2}+\left(\varepsilon k^{2}-k^{2}-2\right) \alpha^{3} e_{3}
$$

and

$$
\Delta \bar{H}=(3 k+\varepsilon k) \alpha^{3} \bar{e}_{2}-\left(\varepsilon k^{2}+k^{2}+2\right) \alpha^{3} \bar{e}_{3},
$$

respectively.

Let $M$ be the flat rotation surface of type $I$ satisfying the condition (6.1). Since $\alpha$ is a non-zero function, from (6.1), (6.2 a) and (6.3 a) we obtain

$$
A e_{3}=(3 \varepsilon k-k) \alpha^{2} e_{2}+\left(k^{2}-\varepsilon k^{2}-2 \varepsilon\right) \alpha^{2} e_{3} .
$$

From this together with (3.3 a) and (3.7 a) we also have

$$
\begin{aligned}
A e_{2}= & \left(2 \alpha \alpha^{\prime}(3 \varepsilon k-k)+\alpha^{2}\left(k^{2}-\varepsilon k^{2}-2 \varepsilon\right)\right) e_{2} \\
& +\left(\alpha^{2}(3 \varepsilon k-k)+2 \alpha \alpha^{\prime}\left(k^{2}-\varepsilon k^{2}-2 \varepsilon\right)\right) e_{3},
\end{aligned}
$$


from which, (3.3 a), (3.7 a) and (6.5) yield

$$
\begin{aligned}
A e_{3}= & \left((3 \varepsilon k-k)\left(2 \alpha^{\prime 2}+2 \alpha \alpha^{\prime \prime}+\alpha^{2}\right)+4 \alpha \alpha^{\prime}\left(k^{2}-\varepsilon k^{2}-2 \varepsilon\right)\right) e_{2} \\
& +\left(4 \alpha \alpha^{\prime}(3 \varepsilon k-k)+\left(k^{2}-\varepsilon k^{2}-2 \varepsilon\right)\left(\alpha^{2}+2 \alpha^{\prime 2}+\alpha \alpha^{\prime \prime}\right)\right) e_{3} .
\end{aligned}
$$

Thus, by combining (6.4) and (6.6) we have

$$
\left\{\begin{array}{l}
(3 \varepsilon k-k)\left(\alpha^{\prime 2}+\alpha \alpha^{\prime \prime}\right)+2 \alpha \alpha^{\prime}\left(k^{2}-\varepsilon k^{2}-2 \varepsilon\right)=0, \\
2 \alpha \alpha^{\prime}(3 \varepsilon k-k)+\left(k^{2}-\varepsilon k^{2}-2 \varepsilon\right)\left(\alpha^{\prime 2}+\alpha \alpha^{\prime \prime}\right)=0,
\end{array}\right.
$$

which imply

$$
\alpha \alpha^{\prime}\left(\left(k^{2}-\varepsilon k^{2}-2 \varepsilon\right)^{2}-(3 \varepsilon k-k)^{2}\right)=0 .
$$

If $\varepsilon=1$, then we have

$$
\alpha \alpha^{\prime}\left(1-k^{2}\right)=0,
$$

from which $\alpha$ is a constant because of (3.8). If we make use of (3.8) again, then we obtain $k=0, r(u)=1$ and $\alpha=1$. Thus the equation (6.3 a) gives $\Delta H=-2 H$, and it is easily seen that the surface $M$ of type $I$ is of 1-type. Furthermore, when $r(u)=1$, the surface is the product of two plane hyperbolas.

Let $\varepsilon=-1$. In the case, we have

$$
\alpha \alpha^{\prime}\left(k^{2}-1\right)^{2}=0,
$$

from this, $\alpha$ is also constant. It follows that $k=0, r(u)=1$ and $\alpha=1$. Therefore, by $(6.2 \mathrm{a})$ and $(6.3 \mathrm{a})$ the surface $M$ of type $I$ is of 1-type.

Similarly, we can prove that the flat rotation surface $M$ of type $I I$ satisfying the condition (6.1) is of 1-type and also the surface $M$ of type $I I$ is the product of a plane circle and a plane hyperbola.

Consequently, we have the following

THEOREM 6.1. Let $M$ be a flat rotation surface of type $I$ or $I I$ in $\mathbb{E}_{2}^{4}$. Then, $M$ is of 1-type if and only if the mean curvature vector $H$ satisfies a partial differential equation

$$
\Delta H=A H, \quad A \in \mathbb{R}^{4 \times 4} .
$$

Combining the our Theorems 3.1, 4.1, 5.1, 6.1 and the main theorems in [23], we have the following 
ThEOREM 6.2 (ChARACTERIZATION). Let $M$ be a flat rotation surface of type $I$ or $I I$ in $\mathbb{E}_{2}^{4}$. Then the following are equivalent:

1. $M$ is of finite type,

2. $M$ is of 1-type,

3. $M$ has finite type Gauss map,

4. $M$ has 1-type Gauss map,

5. $M$ has pointwise 1-type Gauss map,

6. the Gauss map $G$ of $M$ satisfies a partial differential equation $\Delta G=A G$ for some real matrix $A$,

7. the mean curvature vector $H$ of $M$ satisfies a partial differential equation $\Delta H=A H$ for some real matrix $A$,

8. $M$ is either the product of two plane hyperbolas or the product of a plane circle and a plane hyperbola.

\section{References}

[1] L. J. Alías, A. Ferrández, P. Lucas and M. A. Meroño, On the Gauss map of B-scrolls, Tsukuba J. Math. 22 (1998), 371-377.

[2] C. Baikoussis and D. E. Blair, On the Gauss map of ruled surfaces, Glasgow Math. J. 34 (1992), 355-359.

[3] C. Baikoussis, B.-Y. Chen and L. Verstraelen, Ruled surfaces and tubes with finite type Gauss map, Tokyo J. Math. 16 (1993), 341-348.

[4] Surfaces with finite type Gauss map, Geometry and Topology of Submanifolds IV World Scientific Publ. (1992), 214-216.

[5] C. Baikoussis and L. Verstraelen, On the Gauss map of helicoidal surfaces, Rend. Sem. Mat. Messina Ser. II 16 (1993), 31-42.

[6] - On the Gauss map of translation surfaces, Rend. Sem. Mat. Messina Ser. II (in press).

[7] - The Chen-type of the spiral surfaces, Results Math. 28 (1995), 214-223.

[8] B.-Y. Chen, Total Mean Curvature and Submanifolds of Finite Type, World Scientific Publ., 1984.

[9] $\quad$ A report on submanifolds of finite type, Soochow J. Math. 22 (1996), 117-337.

[10] B.-Y. Chen, M. Choi and Y. H. Kim, Surfaces of revolution with pointwise 1-type Gauss map, Submitted for publication.

[11] B.-Y. Chen and S. Ishikawa, On classification of some surfaces of revolution of finite type, Tsukuba J. Math. 17 (1993), 287-298.

[12] B.-Y. Chen and P. Piccinni, Submanifolds with finite type Gauss map, Bull. Austral. Math. Soc. 35 (1987), 161-186.

[13] S. M. Choi, On the Gauss map of surfaces of revolution in a 3-dimensional Minkowski space, Tsukuba J.Math. 19 (1995), 351-367.

[14] _ On the Gauss map of ruled surfaces in a 3-dimensional Minkowski space, Tsukuba J. Math. 19 (1995), 285-304. 
[15] F. Dillen, J. Pas and L. Verstralen, On the Gauss map of surfaces of revolution, Bull. Inst. Math. Acad. Sinica 18 (1990), 239-246.

[16] _ On surfaces of finite type in Euclidean 3-space, Kodai Math. J. 13 (1990), 10-21.

[17] O. J. Garay, On a certain class of finite type surfaces of revolution, Kodai Math. J. 11 (1988), 25-31.

[18] T. Hasanis and T. Vlachos, Surfaces of finite type with constant mean curvature, Kodai Math. J. 16 (1993), 244-252.

[19] C. S. Houh, Rotation surfaces with of finite type, Algebras, Groups and Geometries 7 (1990), 199-209.

[20] D.-S. Kim, Y. H. Kim and D. W. Yoon, Extended B-scrolls and their Gauss map, Indian J. Pure Appl. Math. 33 (2002), 1031-1040.

[21] Y. H. Kim and D. W. Yoon, Ruled surfaces with finite type Gauss map in Minkowski spaces, Soochow J. Math 26 (2000), 85-96.

$[22]$ _ (2000), 191-205.

[23] H. L. Liu, Rotation surfaces with finite type in pseudo-Euclidean space, Algebras Groups Geom. 10 (1993), 253-261.

[24] D. W. Yoon, Rotation surfaces with finite type Gauss map in $\mathbb{E}^{4}$, Indian J. Pure Appl. Math. 32 (2001), 1803-1808.

[25] _ - On the Gauss map of translation surfaces in Minkowski 3-space, Taiwanese J. Math. 6 (2002), 389-398.

Young Ho Kim

Department of Mathematics

College of Natural Sciences

Kyungpook National University

Taegu 702-701, Korea

E-mail: yhkim@knu.ac.kr

Dae Won Yoon

Department of Mathematics Education and RINS

Gyeongsang National University

Chinju 660-701, Korea

E-mail: dwyoon@nongae.gsnu.ac.kr 